\title{
Long-Term Prognosis of Catecholaminergic Polymorphic Ventricular Tachycardia Patients With Ryanodine Receptor (RYR2) Mutations
}

\author{
Masahiro Yamazoe, MD; Tetsushi Furukawa, MD, PhD
}

$\mathbf{C}$ atecholaminergic polymorphic ventricular tachycardia (CPVT) is a malignant inherited arrhythmia syndrome characterized by bidirectional or polymorphic ventricular arrhythmias under conditions of increased sympathetic activity in young patients without structural heart disease. ${ }^{1}$ The common autosomal-dominant form has been linked to mutations in the gene encoding the cardiac calcium-release channel (RYR2). ${ }^{2}$ To date at least 130 unique, almost exclusively missense, mutations in RYR2 have been reported. ${ }^{3}$ A less common but more severe autosomal-recessive form is caused by mutations in the gene encoding cardiac calsequestrin (CASQ2), the major calcium-binding protein in the sarcoplasmic reticulum (SR). ${ }^{4}$ Mutations in these genes have been identified in approximately $60 \%$ of patients diagnosed as CPVT, ${ }^{2}$ and result in inappropriate calcium leakage from the SR, leading to cytosolic calcium overload generating delayed afterdepolarizations, triggered activity, and ventricular arrhythmias, particularly under increased $\beta$-adrenergic tone. CPVT has become recognized as a significant cause of sudden cardiac death in children and young adults. In untreated patients 8-year overall arrhythmic event rates of $58 \%$ and fatal or non-fatal event rates of $25 \%$ have been documented. ${ }^{5} \mathrm{~A}$ history of aborted cardiac arrest, absence of $\beta$-blockers ${ }^{5}$, and carrying recessively inherited CASQ2 mutations $^{6}$ have been reported as risk factors of arrhythmic events.

\section{Article p 1907}

In this issue of the Journal, Kawata and colleagues ${ }^{7}$ evaluate the recurrence rate of fatal cardiac events after initiation of appropriate medical therapy in 34 CPVT patients with RyR2 mutations. During 7.4 years of follow-up, 7 of the 34 patients developed fatal cardiac events, and among them, 6 patients $(85.7 \%)$ were not compliant with exercise restriction or medication therapy. In other words, the majority of CPVT patients were well controlled by appropriate medical therapy, emphasizing the importance of drug compliance and advice to the

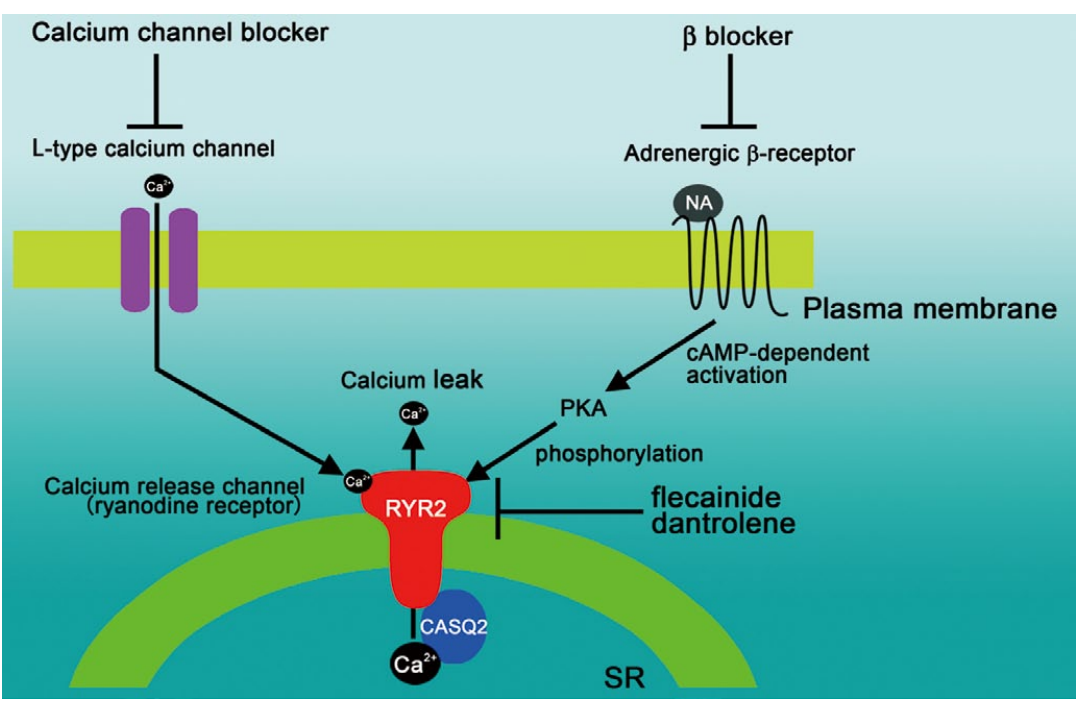

Figure 1. Molecular and signal targets in cardiac myocytes of established and potential medical treatments for catecholaminergic polymorphic ventricular tachycardia. CASQ2, calsequestrin; NA, noradrenaline; PKA, protein kinase A; RYR2, ryanodine receptor 2; SR, sarcoplasmic reticulum.

The opinions expressed in this article are not necessarily those of the editors or of the Japanese Circulation Society.

Received July 26, 2016; accepted July 27, 2016; released online August 12, 2016

Department of Cardiovascular Medicine (M.Y.), Department of Bio-informational Pharmacology, Medical Research Institute (M.Y., T.F.), Tokyo Medical and Dental University, Tokyo, Japan

Mailing address: Tetsushi Furukawa, MD, PhD, Department of Bio-informational Pharmacology, Medical Research Institute, Tokyo

Medical and Dental University, 1-5-45 Yushima, Bunkyo-ku, Tokyo 113-8510, Japan. E-mail: t_furukawa.bip@mri.tmd.ac.jp

ISSN-1346-9843 doi:10.1253/circj.CJ-16-0745

All rights are reserved to the Japanese Circulation Society. For permissions, please e-mail: cj@j-circ.or.jp 


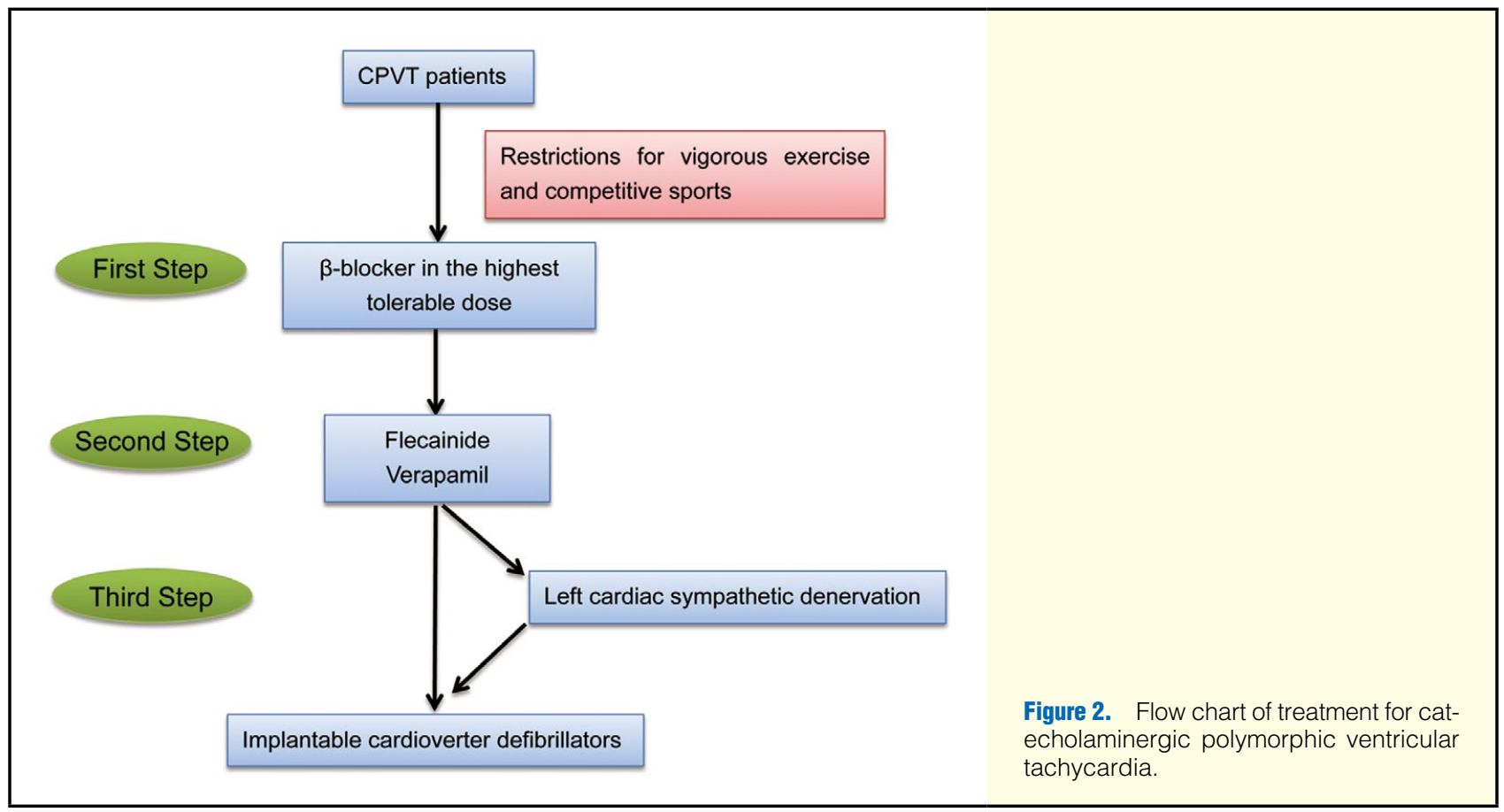

patients and their families about not practicing (competitive) sports.

Figure 1 shows the molecular and signal targets of medical treatment for CPVT. Arguably, $\beta$-blockers are the cornerstone of drug therapy for reducing ventricular arrhythmias during exercise testing and preventing arrhythmic events. ${ }^{8}$ The first step in treating CPVT patients should be a $\beta$-blocker at the highest tolerable dose. Adding a calcium blocker to $\beta$-blocker could be better than $\beta$-blocker alone for preventing exerciseinduced arrhythmias in CPVT. ${ }^{9}$ L-type calcium-channel blockers such as verapamil inhibit the calcium-induced calcium release through RYR2 and also decrease the calcium content of the SR. Although the precise mechanism is not clear, calcium-channel blockers have been shown to be clinically effective in CPVT. ${ }^{9}$ Notably, the antiarrhythmic agent flecainide directly targets the molecular defect in CPVT by inhibiting premature calcium release and triggered beats. ${ }^{10}$ Flecainide is reported to reduce exercise-induced ventricular arrhythmias even in patients not controlled by $\beta$-blocker and/or calcium blocker therapy, and probably plays an irreplaceable role in the treatment of CPVT patients. ${ }^{11}$ In addition, dantrolene, a RYR2 channel inhibitor and effective in malignant hyperthermia, has been shown to prevent exercise- and epinephrineinduced ventricular arrhythmias in CPVT mouse models. ${ }^{12}$ More recently, in CPVT-patient induced pluripotent stem cells-derived cardiomyocytes, dantrolene restored normal calcium spark properties and rescued the arrhythmogenic phenotype. ${ }^{13,14}$ Further clinical trials are needed to confirm the safety and effectiveness in CPVT patients with RyR2 mutations. Left cardiac sympathetic denervation (LCSD) can be performed in patients who are not well controlled by drug therapy. Several case reports and a small case series have shown excellent results of LCSD in severely affected CPVT patients. Finally, implantable cardioverter defibrillators should be recommended for CPVT patients with syncope and/or documented sustained VT despite appropriate medications (Figure 2).

Although the pathophysiological background and appropri- ate treatment are well understood, data for risk stratification are lacking, ${ }^{5}$ particularly for identifying those CPVT patients with a low risk of arrhythmic events in whom treatment can be withheld. Previous studies have shown that genotype-positive relatives demonstrate a wide variety of phenotypes. In a study of 116 drug-free relatives carrying the RYR2 mutation, $50 \%$ had a CPVT phenotype on initial cardiac examination, including $25 \%$ with non-sustained VT. ${ }^{15}$ Interestingly, mutation location may be associated with the severity of phenotype. Relatives with mutations in the C-terminal channel-forming domain of RYR2 had increased odds of non-sustained VT compared with those carrying mutations in the $N$ terminal domain (odds ratio, $4.1 ; 95 \%$ confidence interval, 1.5-11.5). ${ }^{16}$ Similarly, exercise stress testing can be a useful tool for evaluation of asymptomatic relatives of CPVT probands. However, with the present condition, all phenotypically and/or genotypically diagnosed CPVT patients should receive active therapy. Larger cohorts, and multicenter CPVT patient registries are needed to elucidate the exact arrhythmic event rates in different subgroups, as well as the factors that identify individuals at high and low risk, especially in asymptomatic CPVT-associated mutation carriers. Furthermore, the role of genetic and environmental modifiers of the CPVT phenotype ${ }^{17}$ and the genetic background of current genotype-negative cases should be addressed in future studies.

Acknowledgments

None.

\section{Conflict of Interest}

None declared.

\section{References}

1. Sumitomo N. Current topics in cathecolaminergic polumorphic ventricular tachycardia. J Arrhythmia 2015 November 24, doi:10.1016/j. joa.2015.09.008

2. Priori SG, Napolitano C, Memmi M, Colombi B, Drago F, Gasparini 
$\mathrm{M}$, et al. Clinical and molecular characterization of patients with catecholaminergic polymorphic ventricular tachycardia. Circulation 2002; 106: 69-74.

3. Medeiros-Domingo A, Bhuiyan ZA, Tester DJ, Hofman N, Bikker $\mathrm{H}$, van Tintelen JP, et al. The RYR2-encoded ryanodine receptor/ calcium release channel in patients diagnosed previously with either catecholaminergic polymorphic ventricular tachycardia or genotype negative, exercise-induced long QT syndrome: A comprehensive open reading frame mutational analysis. J Am Coll Cardiol 2009; 54: 2065-2074.

4. Postma AV, Denjoy I, Hoorntje TM, Lupoglazoff JM, Da Costa A, Sebillon P, et al. Absence of calsequestrin 2 causes severe forms of catecholaminergic polymorphic ventricular tachycardia. Circ Res 2002; 91: e21-e26, doi:10.1161/01.RES.0000038886.18992.6B.

5. Hayashi M, Denjoy I, Extramiana F, Maltret A, Buisson NR Lupoglazoff JM, et al. Incidence and risk factors of arrhythmic events in catecholaminergic polymorphic ventricular tachycardia. Circulation 2009; 119: 2426-2434.

6. Haugaa KH, Leren IS, Berge KE, Bathen J, Loennechen JP, Anfinsen $\mathrm{OG}$, et al. High prevalence of exercise-induced arrhythmias in catecholaminergic polymorphic ventricular tachycardia mutationpositive family members diagnosed by cascade genetic screening. Europace 2010; 12: 417-423.

7. Kawata H, Ohno S, Aiba T, Sakaguchi H, Miyazaki A, Sumitomo N, et al. Catecholaminergic polymorphic ventricular tachycardia (CPVT) associated with ryanodine receptor (RyR2) gene mutations: Long-term prognosis after initiation of medical treatment. Circ $J$ 2016; 80: 1907-1915.

8. Mizusawa Y. Recent advances in geneticx testing and counseling for inherited arrhythmias. J Arrhythmia 2016 February 5, doi:10.1016/j. joa.2015.12.009.

9. Rosso R, Kalman JM, Rogowski O, Diamant S, Birger A, Biner S, et al. Calcium channel blockers and beta-blockers versus beta-block- ers alone for preventing exercise-induced arrhythmias in catecholaminergic polymorphic ventricular tachycardia. Heart Rhythm 2007; 4: $1149-1154$

10. Watanabe H, Chopra N, Laver D, Hwang HS, Davies SS, Roach DE, et al. Flecainide prevents catecholaminergic polymorphic ventricular tachycardia in mice and humans. Nat Med 2009; 15: 380-383.

11. van der Werf C, Kannankeril PJ, Sacher F, Krahn AD, Viskin S, Leenhardt A, et al. Flecainide therapy reduces exercise-induced ventricular arrhythmias in patients with catecholaminergic polymorphic ventricular tachycardia. J Am Coll Cardiol 2011; 57: 22442254.

12. Kobayashi S, Yano M, Uchinoumi H, Suetomi T, Susa T, Ono M, et al. Dantrolene, a therapeutic agent for malignant hyperthermia, inhibits catecholaminergic polymorphic ventricular tachycardia in a RyR2(R2474S/+) knock-in mouse model. Circ J 2010; 74: 25792584.

13. Jung CB, Moretti A, Mederos y Schnitzler M, Iop L, Storch U, Bellin $\mathrm{M}$, et al. Dantrolene rescues arrhythmogenic RYR2 defect in a patient-specific stem cell model of catecholaminergic polymorphic ventricular tachycardia. EMBO Mol Med 2012; 4: 180-191.

14. Sallam K, Kodo K, Wu JC. Modeling inherited cardiac disorders. Circ J 2014; 78: 784-794.

15. Behere SP, Weindling SN. Catecholaminergic polymorphic ventricular tachycardia: An exciting new era. Ann Pediatr Cardiol 2016; 9: $137-146$

16. van der Werf C, Nederend I, Hofman N, van Geloven N, Ebink C, Frohn-Mulder IM, et al. Familial evaluation in catecholaminergic polymorphic ventricular tachycardia: Disease penetrance and expression in cardiac ryanodine receptor mutation-carrying relatives. Circ Arrhythm Electrophysiol 2012; 5: 748-756.

17. Nakajima T, Kaneko Y, Kurabayashi M. Unveiling specific triggers and precipitating factors for fatal cardiac events in inherited arrhythmia syndromes. Circ J 2015; 79: 1185-1192. 